\title{
Metallic Nano-Rings for Free-Space Extraction of Light from Single Quantum Dots
}

\author{
Oliver J. Trojak ${ }^{1, *}$, Jin Dong Song ${ }^{2}$, Luca Sapienza ${ }^{1}$ \\ ${ }^{1}$ Department of Physics and Astronomy, University of Southampton, SO17 1BJ, UK \\ ${ }^{2}$ Center for Opto-Electronic Materials and Devices Research, Korea Institute of Science and Technology, Seoul 136-791, South Korea \\ o.trojak@soton.ac.uk
}

\begin{abstract}
We show $25 \times$ enhancements of the free-space collection of light from single In/As quantum dots, via metallic nano-rings deposited on the sample surface. Such a lensing effect is broadband, scalable and compatible with any substrate/emitter.

OCIS codes: (300.6280) Spectroscopy, fluorescence and luminescence; (230.3990) Micro-optical devices; (230.5590)

Quantum-well, -wire and -dot devices; (300.6470) Spectroscopy, semiconductors
\end{abstract}

\section{Introduction}

Bright and pure single-photon sources are an essential resource for quantum technology applications. Solid-state single-photon emitters have a number of desirable attributes, which make them suitable for integration into on-chip devices [1]. However, they can suffer from poor extraction efficiencies due to the large refractive index contrast between the bulk semiconductor and air, that results in a small fraction of light from an emitter being collected into free-space.

To address this issue, a number of device geometries have been developed including optical cavities, such as nano-wires, micro-pillars and suspended circular gratings [2]. Such devices have limited wavelength range operation and require fabrication procedures based on deep (micro-pillars and nano-wires) or nanometer accurate etching (gratings). Otherwise, solid immersion lenses deposited on the sample surface allow broadband enhanced extraction of light but can provide modest performances [3].

We propose a new device geometry based on a metallic ring that focuses the excitation light and the emission from a single quantum dot into free-space via a lensing effect [4]. Such rings are deposited around selected single emitters, positioned via a photoluminescence imaging technique [5].

\section{Discussion of the results}

Finite-difference time-domain simulations were performed to optimize the ring dimensions in order to maximize the emission from a dipole source embedded in GaAs material into free space. The sample consists of a GaAs wafer containing a low density $\left(<1000 \mu \mathrm{m}^{-2}\right)$ of InAs/GaAs quantum dots (QDs) grown by molecular beam epitaxy, capped by a $95-\mathrm{nm}$ thick GaAs layer. By using a confocal micro-photoluminescence set up, the light reflected from alignment marks deposited on the sample surface and the photoluminescence emitted by single quantum dots are imaged on an electron-multiplied charge couple device (Fig. 1a). These measurements allow to locate individual QDs with respect to the alignment marks with uncertainties of about $25 \mathrm{~nm}$.
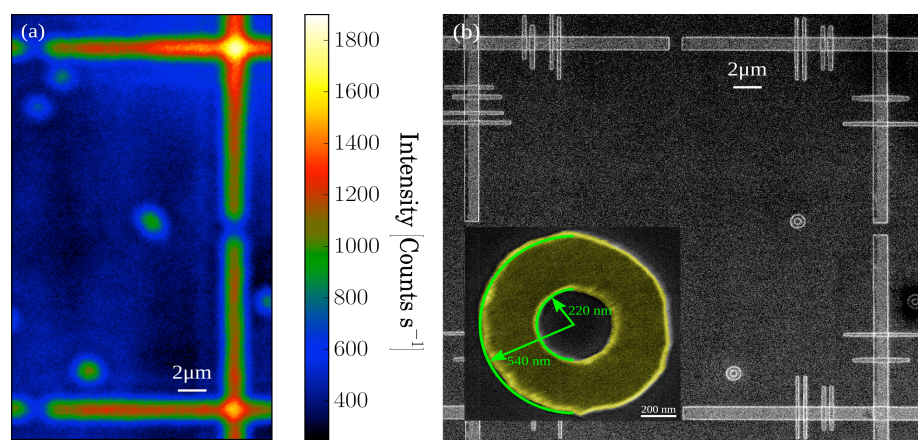

Figure 1: (a) Photoluminescence image showing QDs emission and metallic alignment markers. (b) Scanning electron microscope (SEM) image showing fabricated rings and alignment marks. Inset: SEM of a ring (false color), with its main dimensions overlaid. 


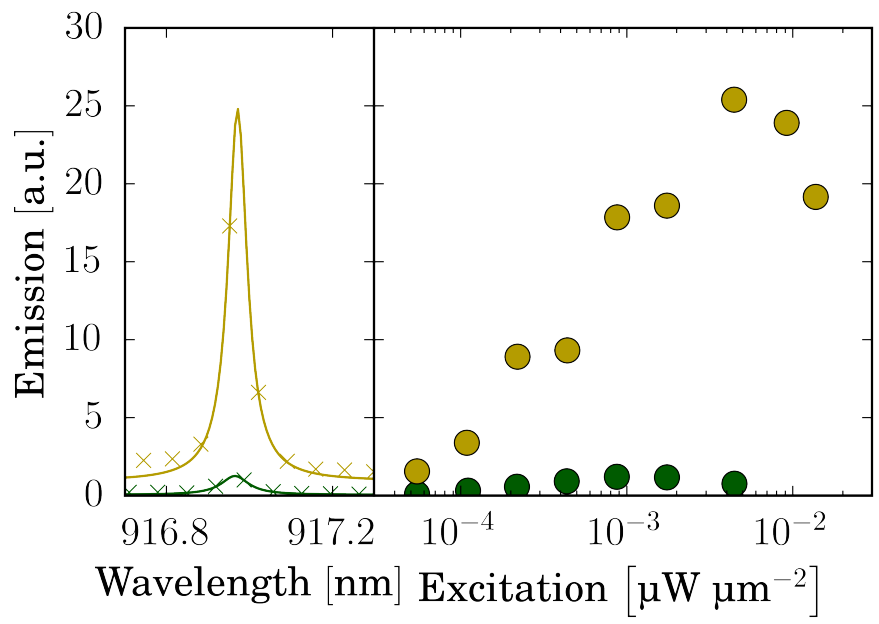

Figure 2: Left panel: Photoluminescence spectra from a single QD collected under $785 \mathrm{~nm} \mathrm{CW}$ laser excitation at QD saturation power, at a temperature of $10 \mathrm{~K}$, before (green) and after (yellow) metallic ring deposition. Right panel: Emission intensity measured as a function of excitation power, for the emission lines shown in the left panels (the same colour coding is used). The intensities are normalised to the saturation level of the lowest intensity peak from the QD in bulk.

Using a $785 \mathrm{~nm}$ continuous-wave laser, individual QDs are excited above-band and the emitted light is spectrally examined with a grating spectrometer. Rings are then deposited on the GaAs surface in correspondence to individual QDs (Fig. 1b), by means of aligned electron-beam lithography, $\operatorname{Cr}(7 \mathrm{~nm}) / \mathrm{Au}(60 \mathrm{~nm})$ deposition, and chemical liftoff.

By measuring the intensity of the light emitted by a QD before and after ring deposition, we evaluate the improvement in the brightness of the light source (Fig. 2). When the metallic ring is deposited on the sample surface and centered in correspondence to the quantum dot position, the light emission is $\sim \times 25$ brighter than the one measured from the same QD in bulk, prior to ring deposition.

\section{Conclusions}

We have demonstrated enhancements in the free-space collection of light emitted by single QDs of up to $\times 25$. The ring device that we have developed is broadband, since it is based on a lensing effect, simple to fabricate and compatible with any combination of substrate and emitter (of classical or quantum light), including defect centers in diamond and in two-dimensional materials, colloidal QDs and quantum wells. The dimensions of the rings make them compatible with photolithography and nano-imprint techniques, thus allowing scalability of the devices. The metallic rings could also be used as top contacts for the application of external electric fields, for charge and/or wavelength tuning, while at the same time increasing the brightness of the light collected into free space.

\section{References}

[1] I. Aharonovich et al., "Solid-state single-photon emitters," Nat. Photon. 10, 631 (2016)

[2] O. Gazzano et al., "Toward optical quantum information processing with quantum dots coupled to microstructures", J. Opt. Soc. Am. B 33, C160 (2016)

[3] M. Sartison et al., "Combining in-situ lithography with 3D printed solid immersion lenses for single quantum dot spectroscopy", Sci. Rep. 7, 39916 (2017)

[4] O. J. Trojak, S. I. Park, J. D. Song, L. Sapienza, "Metallic nanorings for broadband, enhanced extraction of light from solid-state emitters", arxiv.org/abs/1704.07640 (2017)

[5] L. Sapienza et al., "Nanoscale optical positioning of single quantum dots for bright and pure single-photon emission", Nat. Commun. 6, 7833 (2016) 